\title{
BMJ Open A new model of exercise referral scheme in primary care: is the effect on adherence to physical activity sustainable in the long term? A 15-month randomised controlled trial
}

Carme Martín-Borràs, ${ }^{1,2}$ Maria Giné-Garriga, ${ }^{1,2}$ Anna Puig-Ribera, ${ }^{3}$ Carlos Martín, ${ }^{4}$ Mercè Solà, ${ }^{5}$ Antonio I Cuesta-Vargas, ${ }^{6}$ on behalf of the PPAF Group

To cite: Martín-Borràs $C$, GinéGarriga M, Puig-Ribera A, et al. A new model of exercise referral scheme in primary care: is the effect on adherence to physical activity sustainable in the long term? A 15-month randomised controlled trial. BMJ Open 2018;8:e017211. doi:10.1136/ bmjopen-2017-017211

\section{- Prepublication history for} this paper is available online. To view these files, please visit the journal online (http://dx.doi. org/10.1136/bmjopen-2017017211).

Received 8 April 2017 Revised 4 December 2017 Accepted 11 December 2017

Check for updates

For numbered affiliations see end of article.

\section{Correspondence to} PhD Carme Martín-Borràs; mariacarmenmb@blanquerna. url.edu

\section{ABSTRACT}

Introduction Studies had not yet overcome the most relevant barriers to physical activity (PA) adherence. An exercise referral scheme (ERS) with mechanisms to promote social support might enhance adherence to PA in the long term.

Setting A randomised controlled trial in 10 primary care centres in Spain.

Objective To assess the effectiveness of a primary carebased ERS linked to municipal resources and enhancing social support and social participation in establishing adherence to PA among adults over a 15-month period. Participants 422 insufficiently active participants suffering from at least one chronic condition were included. 220 patients (69.5 (8.4) years; 136 women) were randomly allocated to the intervention group (IG) and 202 (68.2 (8.9) years; 121 women) to the control group (CG).

Interventions The IG went through a 12-week standardised ERS linked to community resources and with inclusion of mechanisms to enhance social support. The $\mathrm{CG}$ received usual care from their primary care practice. Outcomes The main outcome measure was self-report PA with the International Physical Activity Questionnaire and secondary outcomes included stages of change and social support to PA practice.

Data collection Participant-level data were collected via questionnaires at baseline, and at months 3, 9 and 15 .

Blinding The study statistician and research assessors were blinded to group allocation.

Results Compared with usual care, follow-up data at month 15 for the ERS group showed a significant increase of self-reported PA (IG: $1373 \pm 1845$ metabolic equivalents (MET) min/week, $n=195 ;$ CG: 919 \pm 1454 MET min/week, $\mathrm{n}=144 ; \mathrm{P}=0.009$ ). Higher adherence (in terms of a more active stage of change) was associated with higher PA level at baseline and with social support.

Conclusions Prescription from ordinary primary care centres staff yielded adherence to PA practice in the long term. An innovative ERS linked to community resources and enhancing social support had shown to be sustainable in the long term.

Trial registration number NCT00714831; Results.

\section{Strengths and limitations of this study}

- This was a large randomised controlled trial to assess the effect and sustainability of a primary care-based exercise referral scheme on prescription enhancing social support and social participation in establishing adherence to physical activity (PA) levels among insufficiently active adult population, over a 15-month period.

- PA interventions were conducted in primary care centres. Primary care is an ideal setting to identify insufficiently active adults, familiarise patients with the importance of doing regular PA, strengthen social support among patients within the same neighbourhood and enhance adherence through community resources.

- PA levels were assessed with a self-report questionnaire, which had shown to be not as accurate and sensitive to changes as an accelerometer. However, more reliable instruments were not available in primary care centres, where the study was conducted.

- Social support and stage of change were also assessed with self-report questionnaires. A mixedmethods design involving discussion groups, would have allowed us to gather relevant information regarding the participants' needs and perceptions.

\section{BACKGROUND}

The major non-communicable diseases such as cardiovascular disease, cancer, stroke and type 2 diabetes have been demonstrably linked to regular physical activity (PA). ${ }^{1}$ Relatively modest amounts of moderate-intensity PA are associated with improved health, physical function and psychological well-being. ${ }^{2}$ Despite the health benefits of regular exercise, it is estimated that $31 \%$ of the global population does not meet current PA recommendations. ${ }^{3}$ In addition to morbidity and premature mortality, physical inactivity is 
responsible for a substantial economic burden worldwide. ${ }^{4}$ The Spanish population is mainly insufficiently active, ${ }^{5}$ which places a huge economic burden on our healthcare system with similar trends worldwide.

As people age, the risk of social isolation and loneliness is increasing. ${ }^{6}$ Observational studies indicate that social capital components are a major protective factor for mental and physical health and mortality. ${ }^{78}$ Social capital interventions, especially those that promote social support and social participation, usually aim at increasing well-being and promoting healthy lifestyles, enhancing sustained health improvements reached within the intervention. Therefore, interventions designed to increase PA levels in the long term and enhance adherence through social support have the potential not only to generate healthcare savings ${ }^{9}$ but also to minimise the severity of multiple chronic diseases, promote the maintenance of function and prevent further loss of independence. ${ }^{10}$

Primary care centres are an optimal setting to enhance $\mathrm{PA},{ }^{11}$ and primary care physicians are the major healthcare providers for people with multiple morbidities. ${ }^{12}$ A high percentage of the population visit their primary care professional at least once a year, ${ }^{13}$ tend to have confidence with them and their suggestions have a high impact in patients' everyday life. ${ }^{14}$ Most interventions based at increasing PA levels had been conducted in primary care settings and there is growing interest in the exercise referral scheme (ERS) model, ${ }^{15}$ in which a member of the primary care team identifies and refers an insufficiently active individual to a third-party service (often a sports centre or leisure facility). This service then prescribes and monitors an exercise programme tailored to the patients' needs.

Although efforts to promote PA through ERS at community level have had some success, ${ }^{13} 15$ the prevalence of insufficiently active individuals remains high. ${ }^{16}{ }^{17} \mathrm{PA}$ is a complex behaviour influenced by demographic, biological, cognitive, emotional, sociocultural and environmental factors ${ }^{18}$; accordingly, individuals face numerous barriers in both the adoption and maintenance of a regular exercise programme. Studies had not yet overcome the most relevant PA barriers to PA adherence, considering intervention fidelity challenges and reduced intervention personalisation when group formats are applied. ${ }^{11}$ Chambers and colleagues ${ }^{19}$ suggested that intervention sustainability relates to the extent that the intervention can continue to be delivered over time and institutionalised within settings, with necessary capacity built to support their delivery. All systems and organisations are faced with the challenge of implementing new practices, yet many of the innovations that are initially successful fail to become part of the habits and routines of the host organisations and communities. However, as Greenhalgh $^{20}$ pointed out in their review, there is a "near absence of studies focusing primarily on the sustainability of complex service innovations."

The main purpose of the study was to assess the effectiveness and sustainability of a primary care-based ERS on prescription enhancing social support and social participation in establishing adherence to PA among insufficiently active adult population, over a 15 -month period.

\section{METHODS}

\section{Study design}

We conducted a two-arm randomised controlled trial comparing the effectiveness of a 12-week PA intervention combined with municipal and sport resources with added social support and social participation strategies, and usual care combined with social education meetings. Study design details are described in a previous publication. The present study reports different outcomes from two additional centres. ${ }^{9}$ Written informed consent was obtained from all subjects. The study was performed in accordance with the Declaration of Helsinki, ethical principles for medical research involving human subjects (64th World Medical Association General Assembly, Brazil, October 2013).

\section{Sample size calculation}

Sample size calculation was estimated for significant changes in PA levels. Four hundred and twenty-four individuals were needed: 212 individuals in the intervention group (IG) and 212 in the control group (CG) in order to detect a difference $\geq 15 \%$ of participants that increased physical activity levels between groups (assuming 50\% in the CG), with a power of $80 \%$ and an alpha level of $0.05 .{ }^{21}$ A dropout rate of $20 \%$ was estimated, based on the pilot study experience. ${ }^{22}$ Four hundred and seventy-six individuals were recruited for the study and out of which 422 participated.

\section{Study population}

Participants were recruited from eight primary healthcare centres (PHCs) in the Barcelona area and surroundings, and two PHCs are located in Malaga. Eligibility criteria included patients of both genders aged 18-85, with at least one chronic disease, independent in rising from a chair and walking with or without a technical aid, who self-reported being insufficiently active, as determined by one question screening tool: 'As a rule, do you do at least half an hour of moderate or vigorous exercise (such as walking, cycling or a sport) on five or more days of the week?'.23

Individuals were ineligible for the study if they were unable to walk, were undergoing an exercise programme, had a diagnosis of severe dementia (not able to understand and/or follow verbal commands), or had had a stroke, hip fracture, myocardial infarction or had undergone hip or knee replacement surgery within the previous 6 months. The methods had been defined in a previous study. ${ }^{9}$

\section{Recruitment and randomisation procedures}

The recruitment process took place in 10 PHCs during the first 3 months of 2009. Until August 2008, 63 


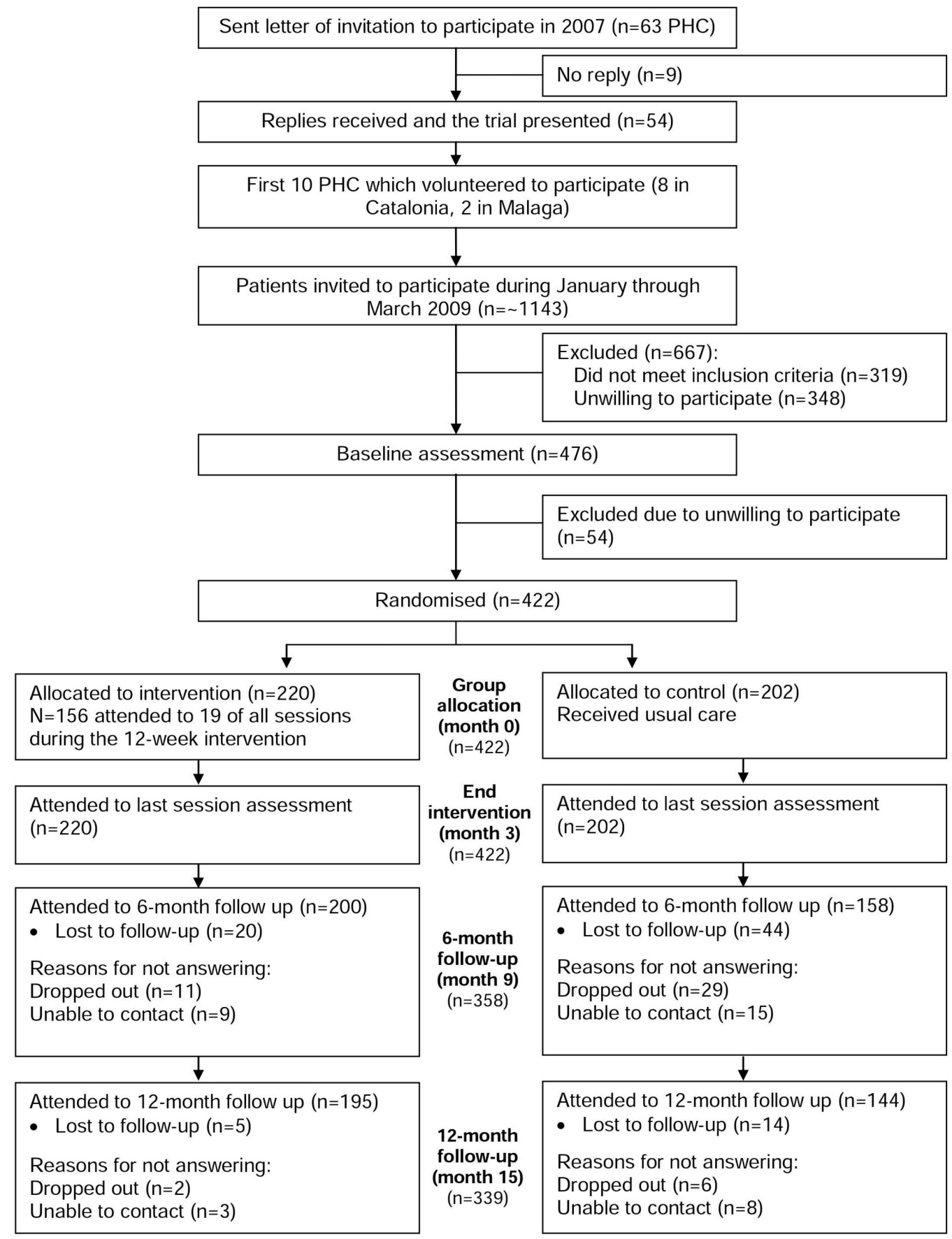

Figure 1 Flow chart of participant's recruitment and trial design. PHC, primary healthcare centre.

randomly selected PHCs in Spain were informed and the trial was presented to the 54 centres which showed interest in participating. Of these, the first 10 centres which volunteered to participate underwent the trial. Two health professionals, who were selected on a voluntary basis from each of the participating centres, were trained. During the recruitment period, the opportunity to participate in the study was offered daily to all patients, who by systematic random sampling were previously identified in the two health professionals' lists. The patients who met the inclusion criteria and agreed to participate were further contacted for an interview and were administered a baseline questionnaire. After baseline measures, they were randomly allocated to the IG or CG, using a centrally generated variable-sized block design. Couples were randomly assigned together. Four hundred and twenty-two subjects participated in the study: 220 patients ( $\mathrm{n}=220 ; 69.5$ (8.4) years; 136 women) were randomly allocated to the ERS (IG), and 220 patients ( $\mathrm{n}=202$; 68.2 (8.9) years; 121 women) were allocated to the CG. The study personnel who maintained the recruitment process and randomisation log were not involved in screening, testing or training procedures. Figure 1 shows the flow chart of participants through the study following the Consolidated Standard of Reporting Trials flow diagram. ${ }^{24}$ 
Table 1 Baseline (month 0) characteristics of intervention and control groups

\begin{tabular}{|c|c|c|}
\hline Variable & $\begin{array}{l}\text { Intervention } \\
(\mathrm{N}=220)\end{array}$ & $\begin{array}{l}\text { Control } \\
(\mathrm{N}=202)\end{array}$ \\
\hline Age (years), mean (SD) & $69.5(8.4)$ & $68.2(8.9)$ \\
\hline Female, n (\%) & $136(61.8)$ & $121(59.9)$ \\
\hline \multicolumn{3}{|l|}{ Anthropometrics } \\
\hline Height (cm), mean (SD) & $158.7(9.6)$ & $159.7(9.4)$ \\
\hline Weight (kg), mean (SD) & $69.3(14.4)$ & $70.1(13.9)$ \\
\hline BMI $\left(\mathrm{kg} / \mathrm{m}^{2}\right)$, mean $(\mathrm{SD})$ & $29.7(4.5)$ & $29.9(4.5)$ \\
\hline HR (bpm), mean (SD) & $81.56(10.2)$ & $80.60(10.5)$ \\
\hline $\mathrm{SBP}(\mathrm{mm} \mathrm{Hg})$, mean (SD) & $140.13(21.9)$ & $147.13(16.9)$ \\
\hline DBP (mm Hg), mean (SD) & $82.25(7.95)$ & $86.53(10.1)$ \\
\hline \multicolumn{3}{|l|}{ Medical conditions } \\
\hline Hypertension, n (\%) & $131(65.5)$ & $111(55)$ \\
\hline Diabetes mellitus, n (\%) & $58(26.4)$ & $51(25.2)$ \\
\hline $\begin{array}{l}\text { Myocardial infarction, } \mathrm{n} \\
(\%)\end{array}$ & $27(12.3)$ & $24(11.9)$ \\
\hline $\begin{array}{l}\text { Congestive heart failure, } \\
\mathrm{n}(\%)\end{array}$ & $21(9.5)$ & $19(9.4)$ \\
\hline $\begin{array}{l}\text { Osteoarticular chronic } \\
\text { problems, } \mathrm{n}(\%)\end{array}$ & 98 (44.5) & $91(45)$ \\
\hline
\end{tabular}

BMI, body mass index; BPM, beats per minute; DBP, diastolic blood pressure; HR, heart rate; SBP, systolic blood pressure.

\section{Outcome measures}

Demographic and health data were collected at baseline (age, blood pressure, resting heart rate, weight, height, body mass index, current medication and associated diseases).

Outcomes were assessed at baseline (month 0), at the end of the intervention (month 3) and at 6-month and 12-month follow-ups after the end of the intervention (months 9 and 15, respectively). Three assistant researchers, blinded to group allocation, obtained all outcomes using telephonic interviews or face-to-face meetings.

The main outcome measure was adherence, means of increasing the number of metabolic equivalents (MET level $\times$ minutes of activityxevents per week) assessed with the short version International Physical Activity Questionnaire. ${ }^{25}$ This instrument has shown good validity and reliability in general population in a previous study.

Secondary outcomes included: (1) attitude towards the PA practice stage of change using the Prochaska scale $(\mathrm{SOC})^{26}$ and (2) social support for PA practice, assessed using the Social Support for Physical Activity Scale (SSPAS) ${ }^{27}$ Reliability and validity of the scale was previously assessed in older adults. ${ }^{27}$

\section{Study treatments}

Intervention group

The intervention was conducted in a primary care available space or nearby outdoor spaces such as public parks. All participants reported to the training facility twice a week for
12 weeks. The PA intervention was group based with 10-15 participants in each group, with no cost to themselves. Each session lasted $60 \mathrm{~min}$, and all protocols incorporated the overload training principle. ${ }^{28}$ All training sessions began with a warm-up (10 min), included an aerobic activity and upper and lower body strength-based exercises $(45 \mathrm{~min}$ ) and ended with cool down with a strong emphasis on social support $(5 \mathrm{~min})$. Standardised sessions were always performed under the supervision of the same PA specialist, previously trained and blinded to the study objectives. During the exercise period, participants were instructed to continue their routine daily activities. Participants were instructed to perform strength training at a perceived exertion intensity of 4-6 (somewhat hard) during the first 2 weeks of training, ${ }^{29}$ without holding their breath during exercises to minimise exercise-induced blood pressure elevations. Training intensity was individually calculated by the $4-8$ repetition maximum method (ie, maximum number of repetitions to failure between 4 and 8) for each exercise using elastic bands and body weight (loaded sit to stand movement). After the familiarisation stage, preferred intensity was established at 6-8 (hard to really hard) of the Borg's scale. This protocol was developed on a pilot training study. ${ }^{22}$

The PA specialist encouraged all participants to conduct a third session each week on their own such as brisk walking. The aim of this third session was twofold: to enhance the autonomy of the participants to meet and share the interest to regular PA practice, and facilitate adherence to PA practice within the group members once the intervention ended. The PA specialist detected a leader in each group to organise these extra sessions and motivate the other participants.

To enhance sustainability of the intervention and adherence to PA practice once the programme ended, all participants were offered a personalised exercise programme with exercises that were performed during the sessions. Moreover, during the last two sessions, visits with all participants were made to the nearest community resources (eg, sport facilities) where the regular PA practice could be continued. Participants were offered a special monthly rate.

During the PA programme, social support and social participation were enhanced. Social support refers to support received (eg, informative, emotional or instrumental) or the sources of the support (eg, friends met in the group) that enhance recipients' self-esteem or provide stress-related interpersonal aid. ${ }^{30}$ The PA programme included the following mechanisms to enhance social support during the cool-down phase of each session: social influence/social comparison (eg, share the appropriateness of the participants' own attitudes, beliefs and behaviours against standards that are avowed and/or modelled by reference group members), social control (eg, explicit attempts of social network members to monitor, encourage, persuade, remind or pressure a person to adopt or adhere to positive health practices: the PA leader reminded each participant the extra-session appointment and the PA 
Table 2 Effects of the intervention on the physical activity levels

\begin{tabular}{|c|c|c|c|}
\hline Variable & IG & CG & $P$ value \\
\hline PA levels (month 0 ) & $(n=220)$ & $(n=202)$ & \\
\hline \multicolumn{4}{|c|}{ MET min/week, mean (SD) } \\
\hline Vigorous PA & $11(104)$ & $12(125)$ & 0.917 \\
\hline Moderate PA & $139(351)$ & $171(533)$ & 0.463 \\
\hline Walk & $583(624)$ & $731(716)$ & 0.030 \\
\hline Total PA & $749(775)$ & $886(876)$ & 0.092 \\
\hline PA levels (month 3 ) & $(n=220)$ & $(n=202)$ & \\
\hline \multicolumn{4}{|c|}{ MET min/week, mean (SD) } \\
\hline Vigorous PA & $172(647)$ & $180(1145)$ & 0.928 \\
\hline Moderate PA & $882(1139)$ & $520(1778)$ & 0.012 \\
\hline Walk & $1362(1594)$ & 967 (988) & 0.003 \\
\hline Total PA & 2416 (2154) & 1666 (2819) & 0.002 \\
\hline PA levels (month 9) & $(n=200)$ & $(n=158)$ & \\
\hline \multicolumn{4}{|c|}{ MET min/week, mean (SD) } \\
\hline Vigorous PA & 109 (489) & $136(705)$ & 0.668 \\
\hline Moderate PA & $516(980)$ & 255 (1364) & 0.034 \\
\hline Walk & 1494 (1598) & 904 (1057) & 0.000 \\
\hline Total PA & 2120 (1994) & $1295(2154)$ & 0.000 \\
\hline PA levels (month 15) & $(n=195)$ & $(n=144)$ & \\
\hline \multicolumn{4}{|c|}{ MET min/week, mean (SD) } \\
\hline Vigorous PA & $48(300)$ & $67(451)$ & 0.636 \\
\hline Moderate PA & $387(1167)$ & $206(788)$ & 0.084 \\
\hline Walk & 937 (956) & 646 (818) & 0.002 \\
\hline Total PA & $1373(1845)$ & 919 (1454) & 0.009 \\
\hline
\end{tabular}

CG, control group; IG, intervention group; MET,

metabolic equivalent; PA, physical activity.

specialist encourage these meetings), self-esteem (eg, beliefs regarding how good, worthy or competent we are in general and through PA practice: at the end of each session participants were asked to summarise their perceived benefits of PA practice and were encouraged to report their satisfactory feelings regarding the help/support received from the other group members), sense of control (eg, participants were encouraged to report how to cope with obligations such as doing regular PA for health once the PA programme ends) and belonging and companionship (eg, strengthen connections to other people with the same goal regarding PA practice is an important source of a sense of belonging, and belonging implies acceptance and inclusion in a group: the PA specialist detected a leader in each group to strengthen connection among participants). Intervention details were detailed in a previous manuscript. ${ }^{9}$

\section{Control group}

Subjects who were randomly assigned to the control group were asked to continue their routine daily activities and received their usual care from their primary care practice whenever it was needed. The control subjects were called once every 4 weeks to minimise dropouts. Researchers followed a standardised script: they asked how participants were feeling, gave them standardised healthy lifestyle advices and were reminded of the following assessment session.

\section{Statistical analysis}

Week 0 (baseline) demographic characteristics were compared between the groups by independent t-test, except for a few cases where required conditions were not satisfied, and Mann-Whitney U test was used as a non-parametric alternative. $\chi^{2}$ tests were used for between-group comparison of categorical variables at baseline. Because of missing data, all longitudinal analyses for variables that were measured at the four time points (months 0, 3, 9 and 15) were performed according to the intention-to-treat principle. For all outcome measures, all the participants who were randomised were analysed; some participants who were lost during a previous follow-up were contacted during the next follow-up and assigned the previous value obtained (last observation carried forward).

The primary focus of the analysis was on the significance of the interaction between group (IG and CG) and time (months 0, 3, 9 and 15). For all outcome measures, adjusted means and SE were calculated and used to compute 95\% CIs. These CIs were adjusted for any between-group differences at baseline, in order to provide a best estimate of the true effect of the intervention.

Our primary outcome measure was self-reported PA level (energy expenditure). A t-test and a repeated measures analysis of variance (ANOVA) were performed to assess any difference between groups. For the secondary outcome measures, a $\chi^{2}$ was performed for the SOC, and a t-test for the SSPA.

All investigators involved in the data analysis were blinded to the treatment assignment. For the statistical

Table 3 Long-term effects of the intervention on the stages of change

\begin{tabular}{lccl}
\hline Variable & IG & CG & P value \\
\hline SOC (month 0) & $(\mathrm{N}=220)$ & $(\mathrm{N}=202)$ & \\
\hline Inactive, $\mathrm{n}(\%)$ & $220(100)$ & $202(100)$ & 0.658 \\
\hline Precontemplation & $20(9.1)$ & $17(8.4)$ & \\
Contemplation & $68(30.9)$ & $69(34.2)$ & \\
\hline Preparation & $132(60)$ & $116(57.4)$ & \\
SOC (month 15) & $(\mathrm{n}=195)$ & $(\mathrm{n}=144)$ & \\
Inactive, $\mathrm{n}(\%)$ & $81(41.5)$ & $129(89.6)$ & 0.000 \\
\hline Precontemplation & $7(3.6)$ & $26(18.1)$ & \\
\hline Contemplation & $20(10.3)$ & $33(22.9)$ & \\
\hline Preparation & $54(27.7)$ & $70(48.6)$ & \\
Active, $\mathrm{n}$ (\%) & $114(58.5)$ & $15(10.4)$ & 0.000 \\
\hline Action & $63(32.3)$ & $14(9.7)$ & \\
\hline Maintenance & $51(26.2)$ & $1(0.7)$ & \\
\hline
\end{tabular}

CG, control group; IG, intervention group; SOC, stage of change. 
Table 4 Long-term effects of the intervention on the social support for physical activity

\begin{tabular}{llll}
\hline Variable & IG & CG & P value \\
\hline SSPA (month 0) & $(\mathrm{n}=220)$ & $(\mathrm{n}=202)$ & \\
\hline Friends, mean (SD) & $8.63(9.04)$ & $9.10(10.15)$ & 0.619 \\
Family, mean (SD) & $12.03(11.34)$ & $12.52(12.65)$ & 0.676 \\
Total, mean (SD) & $20.38(18.70)$ & $21.65(21.05)$ & 0.511 \\
SSPA (month 15) & $(\mathrm{n}=195)$ & $(\mathrm{n}=144)$ & \\
Friends, mean (SD) & $20.87(14.04)$ & $14.59(14.66)$ & 0.000 \\
Family, mean (SD) & $24.41(14.78)$ & $15.67(14.58)$ & 0.000 \\
\hline Total, mean (SD) & $45.27(26.35)$ & $30.26(27.60)$ & 0.000 \\
\hline
\end{tabular}

CG, control group; IG, intervention group; SSPA, social support for physical activity.

analyses, SPSS V.18.0 software was used, and an alpha level of 0.05 was selected.

\section{RESULTS}

\section{Study recruitment and follow-up}

Individual characteristics and compliance with the protocol

Four hundred and twenty-two were randomised: 220 to the PA programme (IG) and 202 to the CG. Participants in the PA programme were required to complete 24 sessions during the 12 weeks of the intervention, and about their compliance (the attendance of each participant at each session) 19 sessions were required; 156 participants in the IG attended $\geq 19$ of the total sessions. There were no adverse events during the study period. At month 3 (end of intervention), all subjects (100\%) were assessed either faceto-face or through telephonic interviews. At month 9, 200 subjects $(90.9 \%)$ from the IG and $158(78.2 \%)$ from the CG were assessed. At month 15, 195 participants (88.7\%) from the IG and 144 (71.3\%) from the CG reported for the assessment session (see figure 1 for more details). At month 0 (baseline), characteristics of the participants are presented in table 1 .

\section{Primary outcome measure}

PA programme participants had greater improvements than those in the CG in self-reported PA level (MET min/ week) at the end of the programme. These improvements were maintained at 9-month and 15-month follow-ups, with significant group-by-time comparisons by the end of the study. The effects of the exercise programme on the primary outcome measure are shown in table 2. Self-reported PA levels showed a decline following cessation of training, but energy expenditure between month 0 and all follow-up measures remained significant. In contrast, individuals in the CG demonstrated no significant changes or decreased their 9-month and 15-month measures with respect to their month 0 values.

Energy expenditure in moderate self-reported PA and walking followed similar patterns and improved significantly $(\mathrm{P}<0.05)$ in the IG from baseline to 3-month measures (end of intervention), and from baseline to 9-month and 15-month follow-ups with no significant change in the CG. These two variables also showed a non-significant decline after cessation of training (9-month follow-up: IG=2120 (1994) MET min/week; $\mathrm{CG}=1295$ (2154); $\mathrm{P}=0.000$ ) (15-month follow-up: $\mathrm{IG}=1373$ (1845) MET min/week; CG=919 (1454); $\mathrm{P}=0.009$ ).

\section{Secondary outcome measures}

A significant $(\mathrm{P}<0.05)$ group effect was established for all 9-month and 15-month measures; for every dependent variable, PA programme performed better than the CG. The effects of the exercise programme on selected secondary outcome measurements are shown in tables 3 and 4. SOC and SSPA measures are only shown for baseline and long-term follow-up (month 15) for a best estimate of the long-term effects.

With respect to SOC, the proportion of participants that moved straight into the doing enough PA stage (action) was higher in the IG at 15 -month follow-up ( $\mathrm{IG}=58.5 \%$; $\mathrm{CG}=10.4$ ), with significant group-by-time comparisons.

With respect to total PA social support, IG participants had greater improvements than those in the CG from baseline to 3-month measure, that also were sustained in the 15-month follow-up (see table 4 ). The family and friends social support improved significantly $(\mathrm{P}<0.05)$ in the PA programme group from baseline to 15 -month follow-up.

\section{DISCUSSION}

The two major findings of this study were that: (1) a PA programme led by physical activity specialists and linked to community resources with enhancement of social support and social participation was effective in sustaining regular PA practice (establishing adherence) in the long term (self-reported PA levels and SOC) and (2) social support was perceived significantly higher in the IG participants in the long term.

Considerable uncertainty remains as to the effectiveness of ERS for improving PA levels or health indicators, or whether they are an efficient use of resources for insufficiently active population with or without a medical diagnosis. ${ }^{11}$ The proportion of individuals achieving 90-150 min of at least moderate-intensity activity per week at 6-12 months' follow-up was greater for ERS than usual care in a recent systematic review assessing the effects of ERS in primary care. ${ }^{31}$ Older patients and those referred for coronary heart disease risk factors appeared to be more likely than others to increase their levels of physical activity. ${ }^{31}$ However, referral to an ERS did not lead to changes in objective measures of health such as weight or blood pressure. ${ }^{31}$

Qualitative evidence suggested that interventions enabling the development of social support networks were beneficial in promoting uptake and adherence. ${ }^{31}$ However, it is not possible to identify what elements of the intervention support successful uptake of ERSs, adherence to ERSs and long-term behaviour change. A 
PA programme conducted in primary care by PA specialists and primary care health professionals could allow insufficiently active patients to familiarise with a regular PA habit and introduce PA practice into their routine activities before referring them to municipal sport-based resources, taking advantage of the confident relationship patients establish with their healthcare providers and the high impact their suggestions have on patients' lives. ${ }^{14}$

Primary care is associated with effective healthcare delivery, ${ }^{32}$ and healthcare systems with strong primary care services such as comprehensiveness of care with the healthcare providers, utilisation and accessibility, have been found to have better quality of care, improved population health and patient satisfaction. ${ }^{32} 33$ A recent study showed that the first contact and comprehensiveness between the patient and their healthcare provider were significantly associated with a better completion of their recommendations. ${ }^{34}$ Thus, appropriate training should be linked to an implementation of an ERS in primary care. The ResearchOne data revealed that total primary care face-to-face consultations increased by $13.3 \%$ between 2010/2011 and 2014/2015, and during the same period, the average patient list size increased by $10 \% .{ }^{35}$ Primary care workload has increased substantially in recent years and it is to be assumed that this trend will continue to increase; thus, primary care might need to refer preventive interventions to community resources. A previously established contact between primary care professionals and PA professionals in different sport facilities and municipal resources might also help overcome a major barrier discussed in a previous study regarding patient's lack of opportunities for sports or leisure activities that conduct healthy PA programmes, ${ }^{36}$ as we suggest in our ERS approach.

ERS use motivating strategies such as social support that have been shown to be an effective tool to increase PA levels in older adults. ${ }^{37}$ However, to achieve improvements in health and well-being associated with physical activity it is necessary to being able to sustain such practice for a long period of time. Approximately half of the participants who initiate an exercise programme will leave the first 6 months. ${ }^{38}$

Evidence had been found regarding the most important facilitators for participants in PA programmes to be underlying social support, perceived health benefits, feeling better and practical aspects of programme location, cost and individually adaptable content. ${ }^{39}$ In this sense, the need to individualise exercise intensity and progression regarding each participant's functional level is twofold: the heterogeneity of most ERS groups could be overcome, and participants would be more challenged and thus, motivated to enhance adherence. ${ }^{39}$

A study showed that the three most frequently cited barriers to engage in a PA programme were poor health $(57.7 \%)$, lack of company $(43.0 \%)$ and lack of interest $(36.7 \%){ }^{36}$ Facilitators to overcome the abovementioned barriers could be the individualisation of the exercise (adaptable content according each participant's physical function) and the enhancement of social support within the programme. Sense of support, belonging, social network and like-mindedness flows over into many of the perceived benefits participants' experience, particularly in terms of well-being. ${ }^{40}$ However, physical health gains and feelings of wellbeing are also necessary to hold people in a programme; as participants grow in physical and general well-being an energising and empowering effect takes place. The appropriate workload should be adapted to maximise gains in health outcomes. ${ }^{41}$

There are some limitations to this efficacy trial. The PA programme aimed to assess long-term adherence to PA practice increasing PA levels from baseline. Measuring PA levels accurately normally involves the overlap of three techniques. These are observational, perceptual (self-report) and some form of objective measurement such as accelerometry or doubly labelled water. ${ }^{42}$ This was beyond the scope of the present study. Unfortunately, it is difficult to measure PA levels objectively in large studies, and our data are based on self-reports. Moreover, contamination in the control group may have occurred, due to exposure of participants in the same primary care centre where the IG conducted the programme, indicated by a weak positive trend in the stages of change instrument. ${ }^{43}$

Social support also was assessed with a self-report questionnaire, limited to collect potentially interesting data. A mixed-methods design, involving discussion groups would allow us to gather relevant information regarding the participants' needs and perceptions.

Related to results, the last observation carried forward approach was used for missing data. This might have caused that the short-term results had been extended over time in those individuals with missing information. As the dropout rates are higher in the CG, they will have estimates closer to the baseline measures compared with the IG participants.

Finally, in our ANOVA, the residuals from physical activity levels are not normally distributed according to Kolmogorov-Smirnov test (results not shown in the manuscript). However, this test in large samples $(n>30)$ does not strictly require ensuring the normality of the residuals. The meaning asymptotically approaches the exact $\mathrm{P}$ values, and ANOVA residuals do not need to be close to normal in order to fit the model.

The Programa de Promoció d'Activitat Física (PPAF) study is an ERS approach conducted in primary care to promote regular PA practice. It provides evidence of its sustainability in the long term linking the physical activity programme to community resources with enhancement of social support and social participation to establish adherence to regular PA practice. In light of our findings, the PPAF intervention should be suitable for clinical practice with important implications in physical activity promotion in primary healthcare. However, the effectiveness of such scheme should be assessed in other settings and targeting other population at risk. 


\section{CONCLUSION}

The ERS intervention showed to be potentially suitable for clinical settings and primary care centres. In summary, our findings indicate that a 12-week PA programme linked to municipal resources and enhancement of social support and social participation and conducted in a primary care setting is a safe, effective and sustainable intervention in insufficiently active adults in maintaining and increase of self-reported PA levels in the long term.

While it is recognised that adherence is multifaceted, incorporating the views of older adults regarding their needs and perceptions when a physical activity-based programme is aimed to be designed, may enhance sustainability of the intervention and adherence in the long term, ${ }^{44}$ as well as enhancing social support and social participation within the programme.

\section{Author affiliations}

${ }^{1}$ Department of Physical Activity and Sport Sciences, Faculty of Psychology, Education and Sport Sciences (FPCEE) Blanquerna, Ramon Llull University, Barcelona, Spain

${ }^{2}$ Department of Physical Therapy, Faculty of Health Sciences (FCS) Blanquerna, Ramon Llull Univesity, Barcelona, Spain

${ }^{3}$ Department of Physical Activity and Sport Sciences, Universitat de Vic, Vic, Spain ${ }^{4}$ Research Unit of Barcelona, Primary Healthcare Research Institution IDIAP Jordi Gol, Barcelona, Spain

${ }^{5}$ Primary Health Center Les Planes, Sant Joan Despí, Barcelona, Spain

${ }^{6}$ Department of Physical Therapy, Universidad de Málaga, Málaga, Spain

Acknowledgements Sincere thanks to all the PHCs that participated in this study and the Research Unit of Barcelona of the Institut Català de la Salut for its collaboration. Many thanks to all professionals who assembled the data, for their positive attitude and unfailing efforts.

Collaborators The members of PPAF Group include José Antonio Losada, Elena Albarrilla, Miquel Muñoz, Eva Castillo, Agustí Guiu, Ana Cascos, Esperanza Fernández, Cristina Martínez, Eulàlia Duran, Maribel Rivera, Cristina Martin, Mònica Castro, Immaculada Roig, Cristina Pérez, M Roser Sánchez, Luz Pastor, Elena Briones, Jose Luis del Val, Miguel Rodríguez-Alcazar, Miguel Jiménez-Grande, Josefa Sampedro-Fernández, María del Carmen Martín-Ruiz.

Contributors CM-B and MG-G: designed the trial and contributed to the funding application. AP-R and CM: helped design the trial and provided general practice expertise. CM-B and MG-G: led the writing of this paper. CM and AIC-V: developed the analysis plan and performed the quantitative analyses. CM and MG-G: were responsible for trial coordination with the support of AP-R, MS, AIC-V and CM with general practices in each primary care centre. MS, AP-R and CM: provided general practice expertise and contributed to the training of general practitioners offering the direct access pathway. AP-R and CM: provided senior trials leadership, expertise and oversight. All authors contributed to this paper and approved the final version.

Funding This work was supported by a research grant from the Catalan Society of General Practice (CAMFIC) (grant no: 2009/010).

Competing interests None declared.

Patient consent Obtained.

Ethics approval Clinical Investigation Ethics Committee of the Primary Care Research Institution Jordi Gol.

Provenance and peer review Not commissioned; externally peer reviewed.

Data sharing statement No additional data are available.

Open Access This is an Open Access article distributed in accordance with the Creative Commons Attribution Non Commercial (CC BY-NC 4.0) license, which permits others to distribute, remix, adapt, build upon this work non-commercially, and license their derivative works on different terms, provided the original work is properly cited and the use is non-commercial. See: http://creativecommons.org/ licenses/by-nc/4.0/ (c) Article author(s) (or their employer(s) unless otherwise stated in the text of the article) 2018. All rights reserved. No commercial use is permitted unless otherwise expressly granted.

\section{REFERENCES}

1. Dishman R, Heath G, Lee IM, et al. Physical activity epidemiology. Champaign, II: Human Kinetics, 2013.

2. Booth FW, Roberts CK, Laye MJ. Lack of exercise is a major cause of chronic diseases. Compr Physiol 2012;2:1143-211.

3. Hallal PC, Andersen LB, Bull FC, et al. Global physical activity levels: surveillance progress, pitfalls, and prospects. Lancet 2012;380:247-57.

4. Ding D, Lawson KD, Kolbe-Alexander TL, et al. The economic burden of physical inactivity: a global analysis of major non-communicable diseases. Lancet 2016;388:1311-24.

5. Ministerio de Sanidad y Consumo. Encuesta nacional de salud. Madrid: Ministerio de Sanidad y Consumo, 2001.

6. World Health Organization. Active ageing: a policy framework. Geneva: World Health Organization, 2002. http://www.who.int/ ageing/publications/active_ageing/en/. (accessed 23 Feb 2014).

7. Ehsan AM, De Silva MJ. Social capital and common mental disorder: a systematic review. J Epidemiol Community Health 2015;69:1021-8.

8. Nyqvist F, Pape B, Pellfolk T, et al. Structural and cognitive aspects of social capital and all-cause mortality: a meta-analysis of cohort studies. Soc Indic Res 2014;116:545-66.

9. Giné-Garriga M, Martin-Borràs C, Puig-Ribera A, et al. The effect of a physical activity program on the total number of primary care visits in inactive patients: a 15-month randomized controlled trial. PLoS One 2013;8:e66392.

10. Lee IM, Shiroma EJ, Lobelo F, et al. Effect of physical inactivity on major non-communicable diseases worldwide: an analysis of burden of disease and life expectancy. Lancet 2012;380:219-29.

11. Pavey T, Taylor A, Hillsdon M, et al. Levels and predictors of exercise referral scheme uptake and adherence: a systematic review. $J$ Epidemiol Community Health 2012;66:737-44.

12. Prados-Torres A, Poblador-Plou B, Calderón-Larrañaga A, et al. Multimorbidity patterns in primary care: interactions among chronic diseases using factor analysis. PLoS One 2012;7:e32190.

13. Grandes G, Sánchez A, Torcal J, et al. Targeting physical activity promotion in general practice: characteristics of inactive patients and willingness to change. BMC Public Health 2008;8:172.

14. Lewis BS, Lynch WD. The effect of physician advice on exercise behavior. Prev Med 1993;22:110-21.

15. Duda JL, Williams GC, Ntoumanis N, et al. Effects of a standard provision versus an autonomy supportive exercise referral programme on physical activity, quality of life and well-being indicators: a cluster randomised controlled trial. Int J Behav Nutr Phys Act 2014:11:10.

16. Buckler A, Servies T; U.S. Preventive Services Task Force. Behavioral counseling interventions to promote a healthful diet and physical activity for cardiovascular disease prevention in adults. Am Fam Physician 2013;87:869-70.

17. Yancey AK, Fielding JE, Flores GR, et al. Creating a robust public health infrastructure for physical activity promotion. Am J Prev Med 2007;32:68-78.

18. Bauman AE, Sallis JF, Dzewaltowski DA, et al. Toward a better understanding of the influences on physical activity: the role of determinants, correlates, causal variables, mediators, moderators, and confounders. Am J Prev Med 2002;23:5-14.

19. Chambers DA, Glasgow RE, Stange KC. The dynamic sustainability framework: addressing the paradox of sustainment amid ongoing change. Implement Sci 2013;8:117.

20. Greenhalgh T. How to spread good ideas: a systematic review of the literature on diffusion, dissemination, and sustainability of innovations in health service delivery and organization, in report for the national co-ordinating centre for nhs service delivery and organisation R \& D (NCCSDO). London: University College, 2004:362.

21. Smitherman TA, Kendzor DE, Grothe KB, et al. State of the art review: promoting physical activity in primary care settings: a review of cognitive and behavioral strategies. Am J Lifestyle Med 2007:1:397-409.

22. Giné-Garriga M, Martin-Borràs $C$. Programa de Promoción de Actividad Física (PPAF) en los centros de atención primaria. Estudio piloto. Atención Primaria 2008;40:374-5

23. Rose SB, Elley CR, Lawton BA, et al. A single question reliably identifies physically inactive women in primary care. $N Z$ Med $J$ 2008;121:U2897. 
24. Schulz KF, Altman DG, Moher D; CONSORT Group. CONSORT 2010 statement: updated guidelines for reporting parallel group randomized trials. Ann Intern Med 2010;152:726-32.

25. Craig CL, Marshall AL, Sjöström M, et al. International physical activity questionnaire: 12 -country reliability and validity. Med Sci Sports Exerc 2003;35:1381-95.

26. Donovan RJ, Jones S, Holman CD, et al. Assessing the reliability of a stage of change scale. Health Educ Res 1998;13:285-91.

27. Sallis JF, Grossman RM, Pinski RB, et al. The development of scales to measure social support for diet and exercise behaviors. Prev Med 1987;16:825-36.

28. Guyton AC, Hall JE. Tratado de fisiología médica. Madrid: Elsevier, 2007.

29. Borg G. Perceived exertion as an indicator of somatic stress. Scand J Rehabil Med 1970;2:92-8.

30. Dumont M, Provost MA. Resilience in adolescents: protective role of social support, coping strategies, self-esteem, and social activities on experience of stress and depression. $J$ Youth Adolesc 1999;28:343-63.

31. Campbell F, Holmes M, Everson-Hock E, et al. A systematic review and economic evaluation of exercise referral schemes in primary care: a short report. Health Technol Assess 2015;19:1-110.

32. Kringos DS, Boerma WG, Hutchinson A, et al. The breadth of primary care: a systematic literature review of its core dimensions. BMC Health Serv Res 2010;10:65.

33. Stange KC, Nutting PA, Miller WL, et al. Defining and measuring the patient-centered medical home. J Gen Intern Med 2010;25:601-12.

34. Aoki T, Miyashita J, Yamamoto Y, et al. Patient experience of primary care and advance care planning: a multicentre cross-sectional study in Japan. Fam Pract 2017;34:206-12.
35. Baird B, Charles A, Honeyman M, et al. Understanding pressures in general practice. London: The King's Fund, 2016.

36. Moschny A, Platen P, Klaassen-Mielke R, et al. Barriers to physical activity in older adults in Germany: a cross-sectional study. Int $J$ Behav Nutr Phys Act 2011;8:121.

37. Hughes SL, Seymour RB, Campbell RT, et al. Best-practice physical activity programs for older adults: findings from the national impact study. Am J Public Health 2009;99:362-8.

38. Dishman RK. The problem of exercise adherence: fighting sloth in nations with market economies. Quest 2001;53:279-94.

39. Biedenweg K, Meischke H, Bohl A, et al. Understanding older adults motivators and barriers to participating in organized programs supporting exercise behaviors. J Prim Prev 2014;35:1-11.

40. Garmendia ML, Dangour AD, Albala C, et al. Adherence to a physical activity intervention among older adults in a post-transitional middle income country: a quantitative and qualitative analysis. $J$ Nutr Health Aging 2013;17:466-71.

41. Hwang CL, Yoo JK, Kim HK, et al. Novel all-extremity high-intensity interval training improves aerobic fitness, cardiac function and insulin resistance in healthy older adults. Exp Gerontol 2016;82:112-9.

42. Vanhees L, Lefevre J, Philippaerts R, et al. How to assess physical activity? How to assess physical fitness? Eur J Cardiovasc Prev Rehabil 2005;12:102-14.

43. Jenum AK, Anderssen SA, Birkeland KI, et al. Promoting physical activity in a low-income multiethnic district: effects of a community intervention study to reduce risk factors for type 2 diabetes and cardiovascular disease: a community intervention reducing inactivity. Diabetes Care 2006;29:1605-12.

44. Farrance C, Tsofliou F, Clark C. Adherence to community based group exercise interventions for older people: A mixed-methods systematic review. Prev Med 2016;87:155-66. 